\title{
The site-specific integration reaction of Listeria phage A118 integrase, a serine recombinase
}

\author{
Sridhar Mandali ${ }^{1}$, Gautam Dhar ${ }^{1,2}$, Nuraly K Avliyakulov ${ }^{1}$, Michael J Haykinson ${ }^{1}$ and Reid C Johnson ${ }^{1,3^{*}}$
}

\begin{abstract}
Background: A large subfamily of serine recombinases contains long polypeptide segments appended to the C-terminal end of the conserved catalytic domain. Members of this subfamily often function as phage integrases but also mediate transposition and regulate terminal differentiation processes in eubacteria. Although a few members of this subfamily have been studied in purified in vitro systems, key mechanistic aspects of reactions promoted by these recombinases remain to be determined, particularly with respect to the functions of the large C-terminal domain.
\end{abstract}

Results: We have developed and characterized a robust in vitro recombination reaction by the Listeria phage A118 integrase, a member of the subfamily of serine recombinases containing a large C-terminal domain. The reaction occurs in a simple buffered salt solution and exhibits a modest stimulation by divalent cations or spermidine and DNA supercoiling. Recombination with purified A118 integrase is unidirectional, being efficient only between attP and attB DNA sites to either join separate DNA molecules (intermolecular recombination) or to generate deletions or inversions depending on the relative orientation of att sites in cis (intramolecular recombination). The minimal attP site is 50 bp but requires only $44 \mathrm{bp}$ of base sequence information, whereas the minimal attB site is $42 \mathrm{bp}$ and requires $38 \mathrm{bp}$ of base sequence information. DNA exchange occurs between the central $2 \mathrm{bp}$ of attP and attB. Identity between these two base pairs is required for recombination, and they solely determine the orientation of recombination sites. The integrase dimer binds efficiently to full att sites, including the attL and attR integration products, but poorly and differentially to each half-site. The large C-terminal domain can be separated from the $\mathrm{N}$-terminal catalytic by partial proteolysis and mediates non-cooperative DNA binding to att sites.

Conclusions: The basic properties of the phage A118 integrase reaction and its substrate requirements have been elucidated. A118 integrase thus joins the handful of biochemically characterized serine integrases that are serving as models for mechanistic studies on this important class of recombinases. Information reported here will also be useful in exploiting this recombinase for genetic engineering.

Keywords: Serine recombinases, Phage integrases, In vitro recombination, Domain structure, Recombination (att) site determinants and specificity

\section{Background}

Programmed DNA rearrangements mediated by sitespecific recombinases mediate a diversity of biological reactions. The Listeria phage A118 integrase, the subject of this report, catalyzes integration and excision of the viral genome into and out of a specific locus within the

\footnotetext{
* Correspondence: rcjohnson@mednet.ucla.edu

${ }^{1}$ Department of Biological Chemistry, David Geffen School of Medicine at UCLA, Los Angeles, CA 90095-1737, USA

${ }^{3}$ Molecular Biology Institute, University of California, Los Angeles, Los Angeles, CA 90095, USA

Full list of author information is available at the end of the article
}

bacterial host chromosome (Figure 1A) [1]. Other sitespecific recombination (SSR) reactions regulate expression of cell surface proteins, promote the transfer of virulence and antibiotic resistance genes, maintain monomeric circular chromosomes for faithful segregation, or resolve transposition intermediates [2]. Well-characterized SSR systems have been exploited for genetic engineering purposes and have thus greatly added to the molecular geneticists' toolbox [3-6]. SSR reactions are often intricately controlled and studies of their regulation have revealed new concepts for nucleoprotein complex assembly as well as DNA enzymology.

\section{Biomed Central}


A

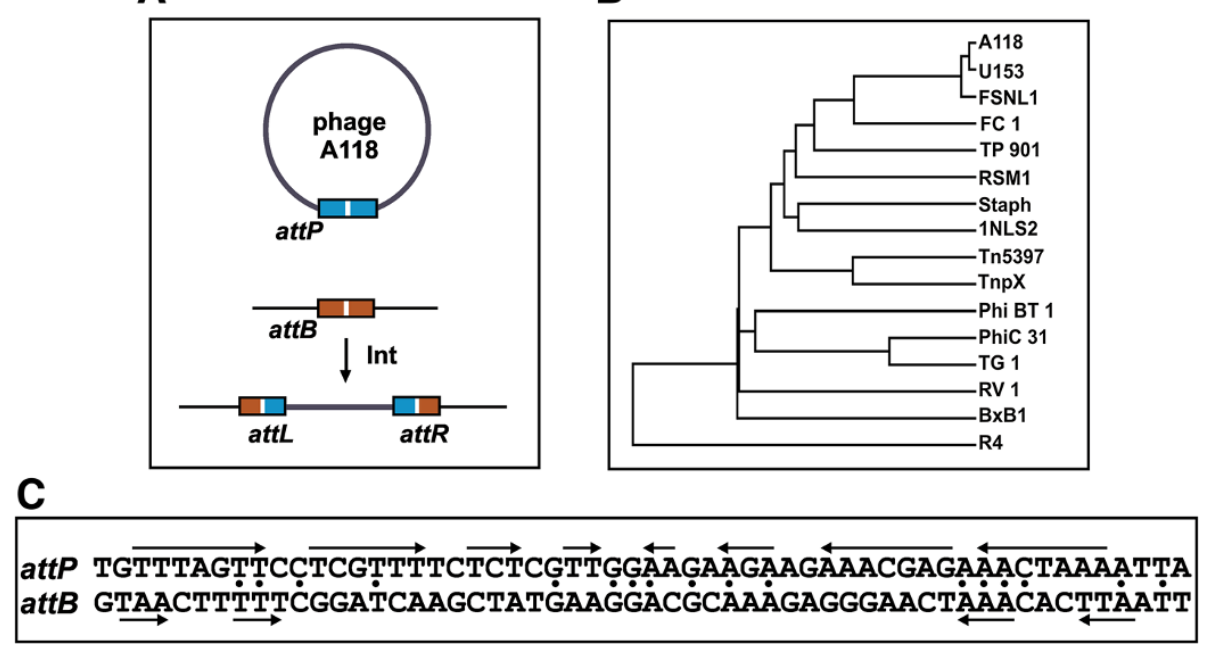

Figure 1 Phage A118 integration reaction and the subfamily of serine recombinases with large C-terminal domains. (A) Schematic representation of phage $A 118$ integrative recombination, where attP on the phage genome recombines with attB within comK in the $L$ isteria monocytogenes chromosome to form the two junction recombination sites attL and attR. (B) Phylogenetic tree of representative serine recombinases with large C-terminal domains (ClustalW2). (C) Sequence (56 bp) surrounding the A118 attP and attB loci. Palindromic sequences within each att site are denoted with arrows, and identical nucleotides between the two recombination sites are marked with dots. We show in this work that the central GG nucleotides define the crossover sites.
Most conservative SSR reactions, where there is no net gain or loss of DNA sequences, fall into two enzymatic families that promote DNA exchange by completely different biochemical mechanisms [7]. Members of the tyrosine recombinase family generate single-strand DNA breaks through the nucleophilic attack of a tyrosine hydroxyl. Two sequential single-strand DNA exchange steps with the formation of a Holliday structure intermediate are required to complete recombination $[8,9]$. By contrast, members of the serine recombinase family generate double strand breaks in DNA through the concerted action of a pair of active site serine residues within the dimeric enzyme. DNA exchange occurs by translocation of subunits that are covalently linked to the cleaved DNA ends within the recombination complex in a reaction known as subunit rotation [10-15].

A118 integrase is a member of the serine recombinase family [16]. Members of this family all share a fairly well conserved 100 to 120 residue catalytic domain followed by a long $\alpha$-helix (referred to as helix $\mathrm{E}$ from resolvase structures), which forms much of the interface between dimer and tetramer forms of the enzyme [7]. Additional domains are appended on the $\mathrm{N}$-terminal or more often C-terminal sides of the catalytic and oligomerization domain. The most intensively studied subfamily of serine recombinases, known as the resolvases and DNA invertases, have a relatively short (typically $<60$ residue) DNA binding domain at their C-terminal end $[17,18]$. Welldeveloped models for orientation-specific DNA site synapsis and DNA exchange have been described for these enzymes that are supported by atomic structures and extensive biochemistry [10,11,15,19-22].

Another subfamily of serine recombinases has much longer C-terminal segments that typically extend 300 to 500 residues [23]. A few members of this group have been investigated in detail, such as the integrases from phages $\phi C 31$, Bxb1, and $\phi R v 1$, and the TnpX transposase from Clostridia [24-27]. The relationship of the integrase sequences from phages A118 and its close relative U153, together with other representatives of this subfamily, is shown in Figure 1B. A feature in common with most of the large $\mathrm{C}$-terminal domains is a relatively centrally located segment with four conserved cysteines [23]; in A118 integrase over one-third of the 41 residues in this segment are also arginine, lysine or histidines. The functional roles of the large C-terminal domain in any of the recombinases remain poorly understood, except that they have been found to specify DNA binding and control aspects of site synapsis in several systems $[24,28,29]$. There is currently no structural information on the large C-terminal domain from any member of the subfamily.

The phage A118 integrase was first described by Calendar and coworkers as part of their analysis of the phage genome sequence [1]. They showed that both A118 and the related U153 phage site-specifically integrate into a Listeria monocytogenes chromosomal locus called $a t t B$ located within the coding region of a gene resembling Bacillus subtilis comK, whose product encodes a transcriptional activator of genes involved in DNA 
uptake [30]. The sequences surrounding the A118 attP recombination site and $a t t B$ are poorly related, and the $a t t B$ region exhibits remarkably little symmetry (Figure $1 \mathrm{C}$ ). Calos and coworkers reported that the A118 integrase together with DNA fragments containing attP and attB can promote recombination in Escherichia coli and mammalian cells, thereby demonstrating its potential for genetic engineering [31].

In this study we characterize the basic biochemical properties of the $\mathrm{A} 118 \mathrm{attP} \times a t t B$ integration reaction using purified integrase. We define the minimal recombination sites and crossover sites and study the binding properties of integrase to the att sites. The properties of the A118 integrase are compared with those of other characterized members of the subfamily of serine recombinases containing large C-terminal domains.

\section{Results}

\section{Purification and partial proteolysis of A118 integrase}

Highly purified preparations of $\mathrm{N}$-terminally histidine tagged A118 integrase were obtained by chromatography through Ni-NTA followed by heparin-Sepharose (Figure 2A, lanes 1 to 5). The activity and tested properties of the His-tagged integrase were indistinguishable from partially purified untagged full-length integrase obtained by heparin-Sepharose chromatography (Figure 2A, lane 6; and data not shown). Because the N-terminal tag has no apparent effect on activity,
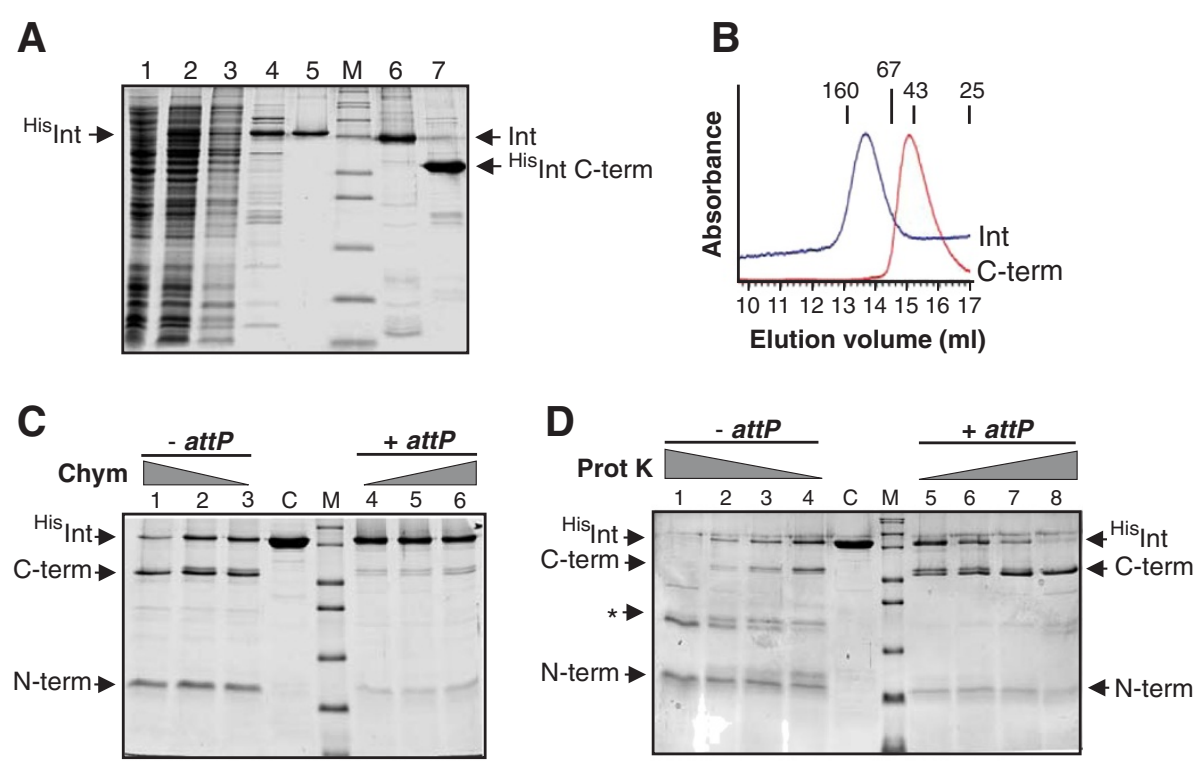

E

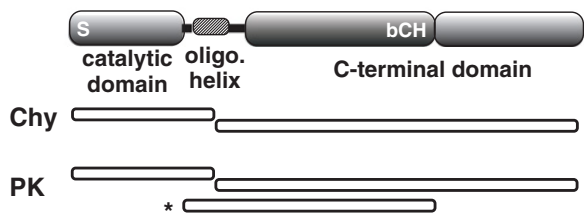

Figure 2 Purification and partial proteolysis of A118 integrase. (A) SDS-PAGE of A118 integrase purification. Lanes 1 to 3, uninduced, induced, and extract after clearing spin, respectively. Lane 4, N-terminally His-tagged integrase after Ni-NTA, and then heparin-Sepharose chromatography (lane 5). Lane 6, native integrase after heparin-Sepharose chromatography, and (lane 7) His-tagged C-terminal domain (residues 158 to 452) after Ni-NTA chromatography. Lane M, molecular weight (MW) markers are 6, 14.4, 21.5, 31, 36.5, 55.4, 66.3, 97.4, 116.3, and 200 kDa.

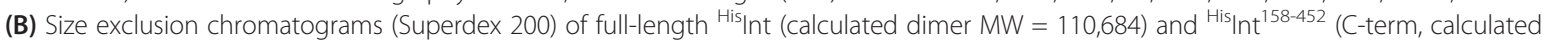
monomer MW = 37,438). Size standards are chymotrypsin $(25 \mathrm{kDa})$, ovalbumin (43 kDa), BSA (67 kDa), and y-globulin 160 (kDa). (C and D) Partial proteolysis of A118 integrase by chymotrypsin and proteinase K, respectively. Integrase ( $3 \mu \mathrm{g}$ ) was digested with 20, 40 , and 60 ng chymotrypsin or $50,75,100$ and $125 \mathrm{ng}$ proteinase $\mathrm{K}$ for $10 \mathrm{~min}$ at $23^{\circ} \mathrm{C}$ in the absence or presence of twofold molar excess of 50 bp attP oligonucleotides, as designated. The products were displayed on a 15\% SDS-PAGE gel. Lane C, no protease control; MW markers (lane M) range from 6 to $66.3 \mathrm{kDa}$, as in (A). (E) Schematic representation of the A118 integrase domain structure based on proteolysis experiments and secondary structure prediction programs. Mutagenesis experiments have shown that the putative active site serine (S) at residue 10 within the catalytic domain is required for recombination. The long putative oligomerization helix is analogous to a-helix $\mathrm{E}$ in resolvase structures. bCH, a basic (11 arginines and lysines) segment between residues 274 and 314 containing four cysteines and four histidines. Chymotrypsin and proteinase $\mathrm{K}$ fragments identified by mass spectrometry are represented below (see text). 
we have employed the more highly purified tagged protein for the studies in this paper. A C-terminal domain fragment beginning at residue 158 was also expressed and partially purified by means of its N-terminal histidine tag (Figure 2A, lane 7). This polypeptide exhibits no catalytic activity but was used for DNA binding studies. Size exclusion chromatography demonstrated that full-length integrase is a dimer in solution, whereas the recombinant $\mathrm{C}$-terminal domain fragment was monomeric (Figure 2B).

Integrase was subjected to limited proteolysis to probe its domain structure. Digestion with chymotrypsin generated two dominant products of approximately 40 and $17 \mathrm{kDa}$ (Figure 2C, lanes 1 to 3). Matrix-assisted laser desorption/ionization time of flight/time of flight (MALDITOF/TOF) mass spectrometry after trypsin digestion of the $40 \mathrm{kDa}$ band generated tryptic peptides extending from integrase residues 143 to 427 and of the $17 \mathrm{kDa}$ band from residues 9 to 129. In a separate experiment, tryptic peptide sequences from the $40 \mathrm{kDa}$ band began at residue 136. Chymotrypsin cleavage at 130 would generate calculated molecular weight products of 16,786 ( 1 to 129 plus His tag) and 38,400 (130 to 452), corresponding to the sizes measured by SDS-PAGE. These data indicate a major chymotrypsin-sensitive site between residues 130 and 136 that separates the small $\mathrm{N}$-terminal catalytic domain from the large C-terminal domain (Figure 2E).

Increasing proteinase $\mathrm{K}$ digestion generated three major products migrating at approximately $40 \mathrm{kDa}, 27$ to $25 \mathrm{kDa}$, and 17 to $15 \mathrm{kDa}$ (Figure 2D). Trypsin digestion and mass spectrometry of the $40 \mathrm{kDa}$ fragment gave the same C-terminal tryptic peptides as the large fragment from the chymotrypsin experiment. The $15 \mathrm{kDa}$ fragment surviving proteinase $\mathrm{K}$ digestion gave four tryptic peptides between residues 9 and 115 (an additional low-intensity peptide corresponding to residues 233 to 246 was also identified). The $27.5 \mathrm{kDa}$ fragment (Figure 2D, asterisk) produced seven tryptic peptides between residues 96 and 331 (calculated molecular weight $=27,823)$. We interpret this fragment to represent a cleavage at the C-terminal end of the catalytic domain and one shortly after the cysteine-histidine rich segment within the C-terminal domain (Figure 2E).

The above proteolysis reactions were all performed without DNA present. We also performed identical experiments in the presence of excess attP DNA. As shown in Figure $2 \mathrm{C}$ (lanes 4 to 6 ), integrase was much more resistant to chymotrypsin cleavage when bound to attP; only a trace of the $\mathrm{N}$-terminal and $\mathrm{C}$-terminal domain products are evident. Cleavage by proteinase $\mathrm{K}$ generating the $\mathrm{N}$-terminal and $\mathrm{C}$-terminal domain products remained relatively robust in the presence of attP DNA, but formation of the $27.5 \mathrm{kDa}$ product was strikingly inhibited (Figure 2D, lanes 5 to 8). These results provide evidence for conformational differences between free and DNA-bound forms of integrase that influence protease accessibility or reactivity.

\section{Intermolecular and intramolecular recombination by $\mathrm{A} 118$ integrase}

Initial experiments testing the activity of purified integrase employed a supercoiled plasmid (pRJ2214) containing attP together with a $100 \mathrm{bp}$ fragment containing $a t t B$ in a reaction analogous to phage integration (Figure 3A). As shown in Figure 3B, linear recombinant products, which were confirmed using radiolabeled attB fragments (data not shown), increased with incubation time. Reaction rates improved with increasing ratios of attB to attP, with maximum rates achieved at a 5:1 molar ratio of linear attB to supercoiled attP $(0.03 \mathrm{pmol})$ with $1 \mathrm{pmol}$ Int (Figure 3C). Subsequent intermolecular reactions employed a 3:1 ratio of short linear to supercoiled plasmid substrates.

We asked whether integrase could catalyze an intramolecular attP $\times a t t B$ deletion reaction as shown in Figure 3D. As shown in Figure 3E (lane 2), Int was efficient at promoting intramolecular deletions between attP and $a t t B$ as revealed by the formation of the two deletion circles, which were each subsequently linearized by restriction enzymes. However, no deletion products were detected in similar intramolecular reactions between $a t t L$ and $a t t R$, the substrates for the excision reaction by phage A118 (Figure 3E, lane 3). Reactions that included L. monocytogenes or E. coli extracts or purified $\mathrm{HU}$ also did not generate detectable attL $\times$ attR deletion products (data not shown). A phage-encoded directionality factor is therefore probably required for the $a t t L \times a t t R$ excision reaction.

\section{In vitro reaction conditions: role of metals, divalent cations, and polyamines}

Reactions were initially performed in $20 \mathrm{mM}$ Tris buffer (pH 7.5), $100 \mathrm{mM} \mathrm{NaCl}, 5 \mathrm{mM}$ spermidine, $5 \mathrm{mM}$ dithiothreitol (DTT), $100 \mu \mathrm{g} / \mathrm{ml} \mathrm{BSA}$, and 5\% glycerol. We individually varied components to evaluate parameters and optimize the in vitro reaction rates using the intramolecular attP $\times a t t B$ deletion reaction. The reaction was optimal over a broad range of $\mathrm{NaCl}$ concentration from 50 to 200 $\mathrm{mM}$, but $\mathrm{KCl}$ or $\mathrm{K}$ glutamate gave up to 20 to $30 \%$ greater reaction than $\mathrm{NaCl}$. Perhaps surprisingly, given the cysteine-rich domain, the reaction was not affected by redox conditions; the absence or presence of up to $10 \mathrm{mM}$ DTT or $5 \mathrm{mM}$ oxidized glutathione had little effect on deletion rates. The presence of $5 \%, 10 \%$, or $15 \%$ glycerol gave 30 to $50 \%$ enhancement and substituting glycerol with ethylene glycol gave 55 to $70 \%$ enhancement. The optimal temperature for the reaction was $30^{\circ} \mathrm{C}$, which was used throughout this work. Experiments employing extracts or purified $\mathrm{HU}$ from L. monocytogenes provided no evidence for a stimulatory co-factor. 
A

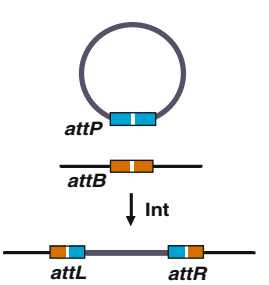

D

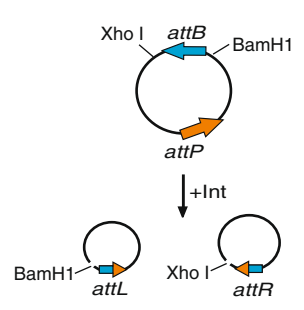

G

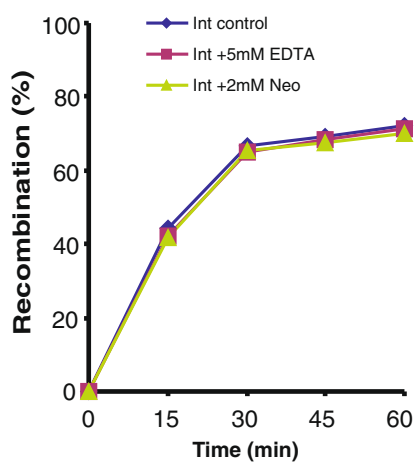

B

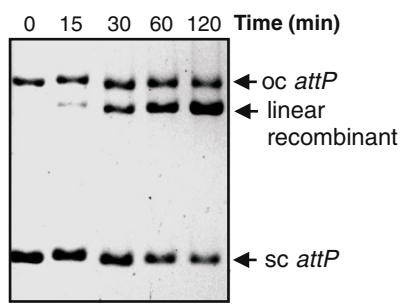

E

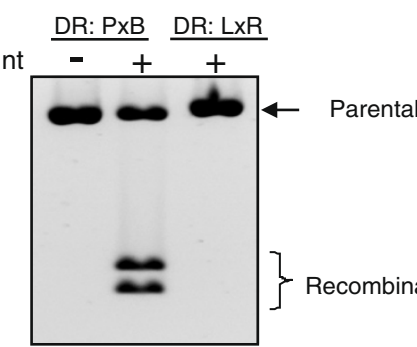

C

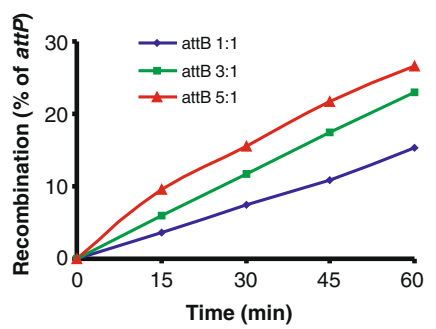

$\mathbf{F}$

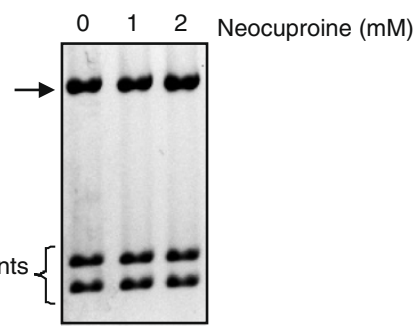

H

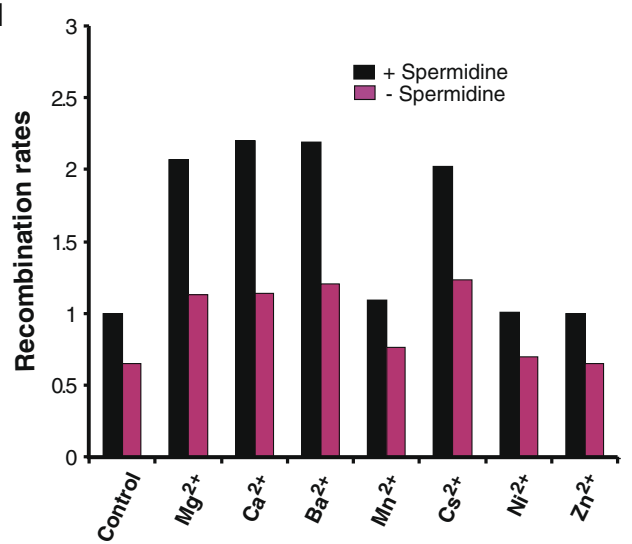

Figure $\mathbf{3}$ In vitro recombination by $\mathbf{A} 118$ integrase. (A) Integrative recombination between a $4.8 \mathrm{~kb}$ supercoiled plasmid containing attP (pRJ2214) and a $100 \mathrm{bp}$ attB fragment generates a $4.9 \mathrm{~kb}$ linear product. (B) Agarose gel showing a time course of intermolecular recombination between supercoiled attP and linear attB substrates. (C) Titration of increasing amounts of the attB fragment relative to the supercoiled attP plasmid on rates of intermolecular recombination. Molar ratios of attB to attP (set at 1) are given. (D) Intramolecular deletion reaction between attP and attB on PBCPB-A1+ generates 3.5 and $3.9 \mathrm{~kb}$ deletion products that are linearized after digestion with BamHI and Xhol, respectively. (E) Deletion reactions between attP and attB (pBCPB-A1+) or between attL and attR (pRJ2913; no recombinant products formed). Integrase reactions were for 40 minutes. (F) Deletion reactions (attP×attB) performed in the absence or presence of the strong metal chelator neocuproine. Integrase reactions were for 40 minutes. (G) Recombination rates in the presence of chelators. Deletion reactions were performed using integrase from storage buffer with $0.1 \mathrm{mM}$ ethylenediamine tetraacetic acid (control, dark blue) or pre-incubated at $4^{\circ} \mathrm{C}$ overnight with $50 \mathrm{mM}$ EDTA (red) or 20 $\mathrm{mM}$ neocuproine (green) in buffer without added metal. Final concentrations of EDTA and neocuproine in the reactions were 5 and $2 \mathrm{mM}$, respectively. $\mathbf{( H )}$ Recombination rates in reactions supplemented with metals and polyamines. Supplements were added to 10-minute deletion reactions with or without $5 \mathrm{mM}$ spermidine. Supplements $\left(\mathrm{MgCl}_{2}, \mathrm{CaCl}_{2}, \mathrm{BaCl}_{2}, \mathrm{MnCl}_{2}, \mathrm{CsCl}\right.$, and $\left.\mathrm{NiCl}_{2}\right)$ were at 10 mM except for $\mathrm{ZnSO}$, which was at $1 \mathrm{mM}$ because higher amounts were inhibitory; $\mathrm{FeCl}_{2}, \mathrm{CoCl}_{2}, \mathrm{CuCl}_{2}$ at $1 \mathrm{mM}$ were inhibitory. Recombination rates (\% deletions in 10 minutes, average of at least three experiments) are given relative to the unsupplemented reaction (control) in the presence of spermidine, which was set to 1.

фC31 integrase was recently reported to contain zinc, which is believed to be associated with its cysteine-rich motif, and the binding of zinc was shown to be functionally important for binding to DNA and recombination [32]. However, other serine integrases promote recombination efficiently in vitro without an apparent requirement for zinc [27,33-35]. Our experiments provide no evidence for a functional role of zinc for A118 integrase. Addition of up to $10 \mathrm{mM}$ ethylenediamine tetraacetic acid (EDTA), $8 \mathrm{mM}$ cyclohexylenedinitrilotetraacetic acid, $10 \mathrm{mM}$ ethylene glycol tetraacetic acid, or $2 \mathrm{mM}$ neocuproine (2,9-dimethyl1,10-phenanthroline) to reactions containing spermidine 
had no inhibitory effect on $a t t P \times a t t B$ deletion rates. In the experiment shown in Figure 3G, A118 integrase was incubated overnight at $4^{\circ} \mathrm{C}$ with $50 \mathrm{mM}$ EDTA or $20 \mathrm{mM}$ neocuproine and the treated enzyme was added to the reaction to give a final concentration of $5 \mathrm{mM}$ EDTA or 2 $\mathrm{mM}$ neocuproine. No differences with the untreated control in the rate of product accumulation were evident. Addition of up to $1 \mathrm{mM}$ zinc to reactions without chelators present had no stimulatory effect. Integrase was also expressed in Luria-Bertani broth (LB) supplemented with a mixture of inorganic micronutrients including $0.5 \mathrm{mM}$ zinc [36] and purified by Ni-affinity chromatography with zinc in all buffers including the storage buffer. The yield and activity of this preparation was indistinguishable from standard integrase preparations.

The effects of divalent cations and metals and their relationship with spermidine on the A118 integrase reaction are summarized in Figure $3 \mathrm{H}$. The presence of spermidine resulted in a $50 \%$ increase in reaction rates. Addition of $\mathrm{Mg}^{2+}, \mathrm{Ca}^{2+}, \mathrm{Ba}^{2+}$, or $\mathrm{Cs}^{+}$in the absence or presence of spermidine gave up to twofold increases in rates but $\mathrm{Mn}^{2+}, \mathrm{Ni}^{2+}$, or $\mathrm{Zn}^{2+}$ had no measurable effects. Int binding to attP DNA fragments by gel mobility shift assays showed no differences in binding affinities when EDTA, $\mathrm{MgCl}_{2}$, or $\mathrm{ZnSO}_{4}$ were included in the binding reactions and electrophoresis buffers (data not shown). These data provide no evidence for an important role for a metal in DNA binding or catalysis by the A118 integrase. The small enhancements of the reaction by additives such as $\mathrm{Mg}^{2+}, \mathrm{Ca}^{2+}, \mathrm{Ba}^{2+}$, and spermidine may be due to global effects on DNA structure $[37,38]$.

\section{DNA substrate specificity: att sites and DNA topology}

A series of experiments were performed to assess the effects of substrate topology and att site location on the A118 integrase reaction. In general, relatively small differences in reaction rates were observed comparing intermolecular versus intramolecular attP $\times a t t B$ reactions on substrates with different topologies. Intermolecular integration reactions were reproducibly slightly more efficient when attP was located on a supercoiled plasmid and $a t t B$ was located on a $100 \mathrm{bp}$ linear fragment than when $a t t B$ was on the plasmid and attP was on the linear fragment (Figure 4A). A comparison of intermolecular reactions between two supercoiled plasmids versus one supercoiled ( $a t t P$ or $a t t B)$ plasmid and one linear $(a t t B$ or $a t t P$ ) plasmid showed little differences in rates or yields, but recombination rates between two linear plasmids were reduced by about 50\% (data not shown). The intramolecular $a t t P \times a t t B$ deletion reaction exhibited about threefold greater reactions rates than the intermolecular supercoiled $a t t P \times$ linear $a t t B$ reaction (Figure $4 \mathrm{~B}$ ), presumably because of a greater frequency of att site collisions when located in cis [39]. DNA supercoiling enhanced the intramolecular deletion reaction by about threefold (Figure 4C), which can also be rationalized by an increase in att site collisions generating productive synapses. There was no measurable difference in deletion or inversion rates between $a t t P$ and $a t t B$ sites separated by $3 \mathrm{~kb}$ in a direct or inverted orientation, respectively, on a supercoiled plasmid (Figure 4D).

Although integrase alone cannot catalyze attL $\times a t t R$ recombination (Figure 3E), we asked whether recombination could occur between $a t t P$ or $a t t B$ and each of the other att sites (Figure 4E,F). Recombination can occur between two attP sites, but the rate is $<5 \%$ that of the $a t t P \times a t t B$ reaction. By contrast, two $a t t B$ sites do not support detectable recombination and no reactions are observed between $a t t L$ or $a t t R$ and $a t t P$ or $a t t B$. We conclude that the A118 integrase exhibits high specificity for the different att sites with only attP $\times a t t B$ functioning effectively.

A118 and U153 integrases from L. monocytogenes phages are very similar in sequence, sharing 95\% amino acid identity (Figure 1B). Moreover, in vivo experiments have shown that phages A118 and U153 lysogenize at the same $a t t B$ locus and that their integrases complement each other [30,31]. The two attP loci are identical within their left half-sites but have five sequence differences on their right half-sites (Figure $4 \mathrm{H}$ ). We find that the A118 integrase will catalyze a low level of integration in vitro between a supercoiled plasmid containing $a t t P^{U 153}$ and linear $a t t B$ but that initial rates and final product yields are threefold to fourfold less than with attP $P^{A 118}$ (Figure 4G; and data not shown). A118 integrase binds to attP $P^{U 153}$ with similar affinity as attP $P^{A 118}$ (data not shown), so the reduced recombination efficiency with attP $P^{U 153}$ cannot be explained by inefficient binding of the integrase to its noncognate attP site.

\section{Binding of A118 integrase to att sites}

Binding of A118 integrase to ${ }^{32} \mathrm{P}$-labeled $100 \mathrm{bp}$ fragments containing attP, attB, attL, and $a t t R$ was evaluated using gel mobility shift assays. As shown in Figure 5A, increasing amounts of integrase were incubated with the attP probe, and the reaction mixture was subjected to electrophoresis in native polyacrylamide gels. The dominant complex contains a dimer of integrase with only a trace of a complex containing an integrase monomer detectable. The binding properties of $a t t B$, as well as $a t t L$ and $a t t R$, are similar to $a t t P$ with apparent $K_{d}$ values for all sites ranging from 48 to $75 \mathrm{nM}$ (Figure 5A). Integrase thus binds efficiently to $a t t L$ and $a t t R$, even though reactions employing these substrates are not productive for recombination.

A118 integrase that had been incubated with increasing amounts of chymotrypsin under conditions similar to those in Figure $2 \mathrm{C}$ was employed in gel mobility shift assays with attP in Figure 5E (lanes 3 to 5). The partially proteolyzed Int generated a single additional complex of 


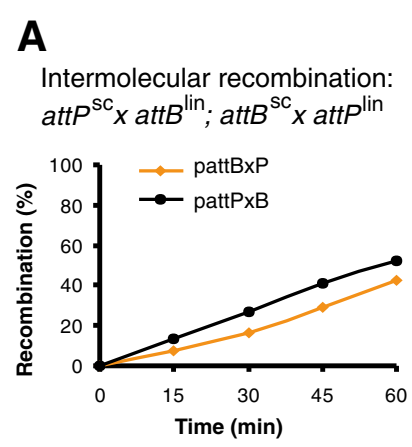

C

Intramolecular recombination: supercoiled vs. linear

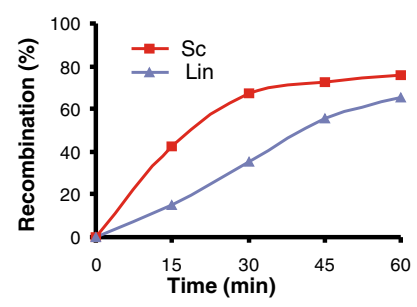

E

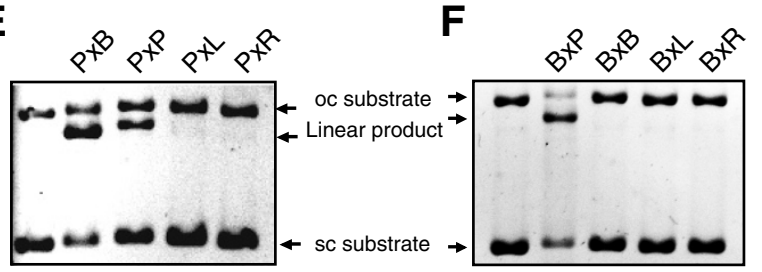

H

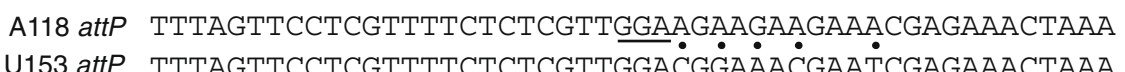

Figure 4 Substrate topology and att site specificity. (A) Comparison of intermolecular integration reactions between supercoiled attP (pRJ2214) and linear attB (100 bp, threefold molar excess over attP) and between supercoiled attB (pRJ2215) and linear attP (100 bp, threefold molar excess over attB) substrates. (B) Comparison of intramolecular attPxattB deletion reactions (pBCPB-A1+) and intermolecular integration reactions between supercoiled attP (pRJ2214) and linear attB. (C) Comparison of intramolecular attPxattB deletion reactions on supercoiled and linear pBCPB-A1+. (D) Comparison of intramolecular attPxattB deletion (pBCPB-A1+) and inversion (pRJ2799) reactions. These two supercoiled substrates are identical except for the relative orientation of att sites. (E) Intermolecular integration reactions (40 minutes) between supercoiled attP (pRJ2214) and $100 \mathrm{bp}$ linear fragments containing different att sites at threefold molar excess over attP. (F) Intermolecular integration reactions (40 minutes) between supercoiled $a t t B$ (pRJ2214) and 100 bp linear fragments containing different att sites at threefold molar excess over attB. (G) Intermolecular integration reactions between supercoiled attP from phage U153 (pRJ2289) and 100 bp linear fragments containing the $a t t B$ site that is used by both phages. $(\mathbf{H})$ Sequence of the A118 and U153 attP sites. Non-identities on the P' sides are highlighted with dots, and the three central nucleotides in common with attB are underlined.

intermediate mobility with that of the full-length dimer complex (lane 2). The migration of this complex corresponds to the migration of the DNA complex formed with a recombinant preparation of the C-terminal domain from residues 158 to 452 (Figure $5 \mathrm{E}$, lane 7; see also Figure 2A, lane 7). The C-terminal domain is therefore sufficient to specify DNA binding activity.
The binding properties of the isolated C-terminal domain were then evaluated (Figure 5F). Unlike full-length integrase, the C-terminal domain predominantly generates Complex I on $a t t P$ and $a t t B$ that would correspond to the binding of a single protomer. Complex II, which forms inefficiently on both substrates, probably contains two protomers. The C-terminal domain binds attP much 


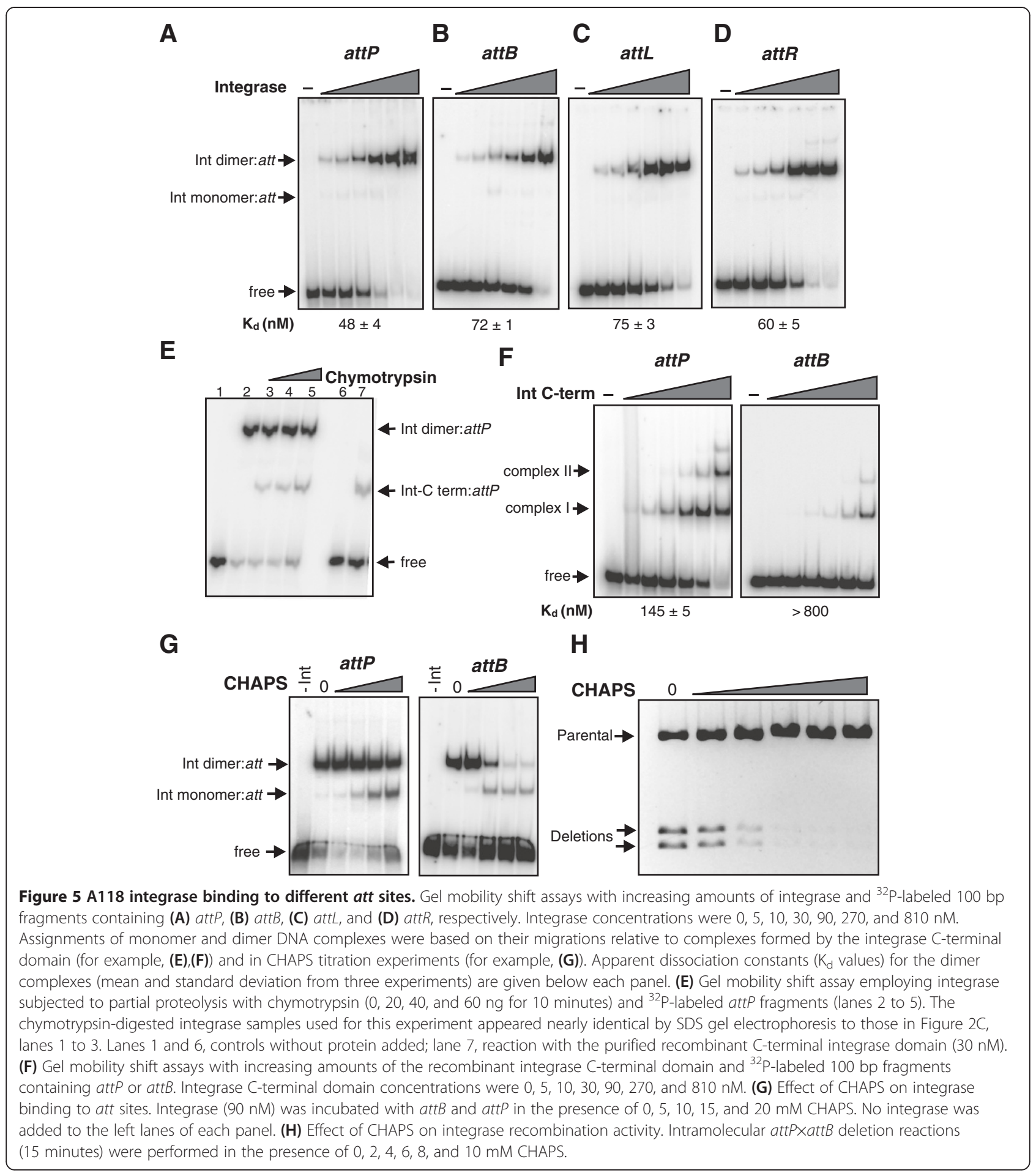

more efficiently than $a t t B$. This contrasts with full-length integrase, which exhibits only 1.5 -fold better binding to attP over $a t t B$ (Figure 5A,B). There is no evidence for cooperative binding by the $\mathrm{C}$-terminal domain, unlike the full-length integrase that binds as a highly cooperative dimer (elaborated further below).
The zwitterionic detergent CHAPS has been shown to specifically destabilize the Hin recombinase dimer [40,41]. We asked how CHAPS affects A118 integrase binding to attP and attB. As shown in Figure 5G, the presence of CHAPS around its critical micelle concentration enhances monomeric binding of full-length 
integrase to both $a t t P$ and $a t t B$. Integrase binding to attB is particularly sensitive to CHAPS as dimer binding is strongly inhibited and the monomeric form predominates above $10 \mathrm{mM}$ detergent. Likewise, CHAPS above 4 $\mathrm{mM}$ has a strong inhibitory effect on recombination activity (Figure $5 \mathrm{H}$ ). These data are consistent with CHAPS destabilizing the oligomerization interface of the A118 integrase, which disturbs binding cooperativity and inhibits formation of a recombinationally active synaptic complex. The nonionic detergent Triton X-100 has no significant effect on integrase binding or recombination at levels above its critical micelle concentration (data not shown).

\section{Integrase binding to half-att sites}

The differential effects of CHAPS on attP and attB by full-length Int, combined with the avid binding of a single $C$-terminal domain protomer to $a t t P$ relative to $a t t B$, suggest that Int does not bind to the two att sites in an equivalent manner (Figure 5). To evaluate this further, binding by the full-length Int and the C-terminal domain to each of the four att half-sites (see below) was probed. The attP sequence is designated PoP' and the attB sequence is designated BoB' to represent the left and right half-sites and central crossover (o) regions as written in Figure 1B. Full-length Int bound predominantly as a dimer $\left(\mathrm{K}_{\mathrm{d}} \sim 1 \mu \mathrm{M}\right)$ and the $\mathrm{C}$-terminal domain exhibited weak monomer binding to the attP Po half-site, but no detectable binding to the oP' half-site (Figure 6A,B,C). Fulllength Int exhibited weak dimer binding $\left(K_{d}>1 \mu \mathrm{M}\right)$ to attB Bo, but again no binding was detectable to the right oB' half-site (Figure 6D,E,F). Dimeric binding to the left half-sites, even in the case for an attP probe where no DNA was present over the right half-site (data not shown), confirms the strong dimer cooperativity with fulllength Int. These results also show that the two half-sites of $a t t P$ and $a t t B$ are functionally distinct since neither the P' nor B' half-sites exhibit detectable binding.

\section{The minimal attP and attB sequence}

The minimal sequence requirements for $a t t P$ and $a t t B$ function were determined both in vitro and in vivo. The minimal sequence length for attP was determined in vitro by intermolecular integration reactions between supercoiled pRJ2215 containing a $100 \mathrm{bp}$ attB segment and a series of duplex oligonucleotides of decreasing lengths representing the attP sequence (Figure 7A,C). A synthetic duplex of $50 \mathrm{bp}$ covering the imperfect palindromic sequence surrounding the attP crossover point supported recombination with similar efficiency to a 100 bp attP fragment. However, a 48 bp duplex that eliminated the outer base pairs exhibited no detectable recombination in vitro.
Recombination proficiency of the resected attP sites in vivo was evaluated by intramolecular deletion formation in E. coli using plasmids derived from pBCPB-A1+ [31]. The attP region was replaced with the resected attP segments and transformed into a recA $\triangle l a c$ strain containing a compatible plasmid (pIntA1) expressing integrase. Deletion removes the lacZ gene between attP and $a t t B$, resulting in white colonies on X-gal media (Figure 7E). We found that attP segments as small as 44 bp remained competent for in vivo recombination, but a $42 \mathrm{bp}$ attP was inactive (Figure 7A). These results suggest that while a minimum of $50 \mathrm{bp}$ of DNA is required for $a t t P$ function, specific base sequence information is only required within the $44 \mathrm{bp}$ sequence. In support of this interpretation, in vitro deletion reactions with the plasmid containing $44 \mathrm{bp}$ attP sites were as efficient as those with $50 \mathrm{bp}$ (data not shown).

The attB site was dissected in a similar manner. In vitro assays with attB duplexes of decreasing length showed that 41 bp was minimally sufficient for recombination, although rates were less than $15 \%$ of the longer substrates (Figure 7B,D). In vivo deletion assays (Figure 7B), as well as in vitro reactions on the deletion plasmid substrates (data not shown), showed that sequence information within a $37 \mathrm{bp}$ region was required. We conclude that a 42 to $44 \mathrm{bp}$ segment is minimally required for full $a t t B$ function with specific base sequence information needed over a 36 to $38 \mathrm{bp}$ region. The length of the $a t t B$ site is thus 6 to 8 bp shorter than attP.

\section{Identification of the crossover site}

There are only $3 \mathrm{bp}$ in common between the centers of $a t t P$ and $a t t B$ (Figure 7A), implicating the crossover region to be within this segment [1]. To precisely define the crossover site, we individually changed each of these positions in $a t t B$ and evaluated their ability to support intramolecular deletion reactions with attP-wt in vitro and in vivo (Figure 8B,C). Changes at G1 or G2 abolished recombination whereas $\mathrm{A} 3 \mathrm{~T}$ had no effect on recombination efficiency, implicating the GG dinucleotide as the crossover segment. To confirm this assignment, we made the same changes in $a t t P$ and measured recombination between mutant attP and attB sites. The double mutants with identical changes at G1 or G2 were active for recombination in vitro (Figure 8D) and generated about $50 \%$ recombinant transformants (white colonies) in vivo (Figure 8E).

\section{Orientation of att sites is distinguished solely by the $2 \mathrm{bp}$ crossover region}

In other serine recombinase systems, recombination site orientation is solely determined by the asymmetry of the two core nucleotides at the crossover site. In the case of the $\mathrm{G} 1 \mathrm{C}$ or $\mathrm{G} 2 \mathrm{C}$ mutants the core nucleotides are 

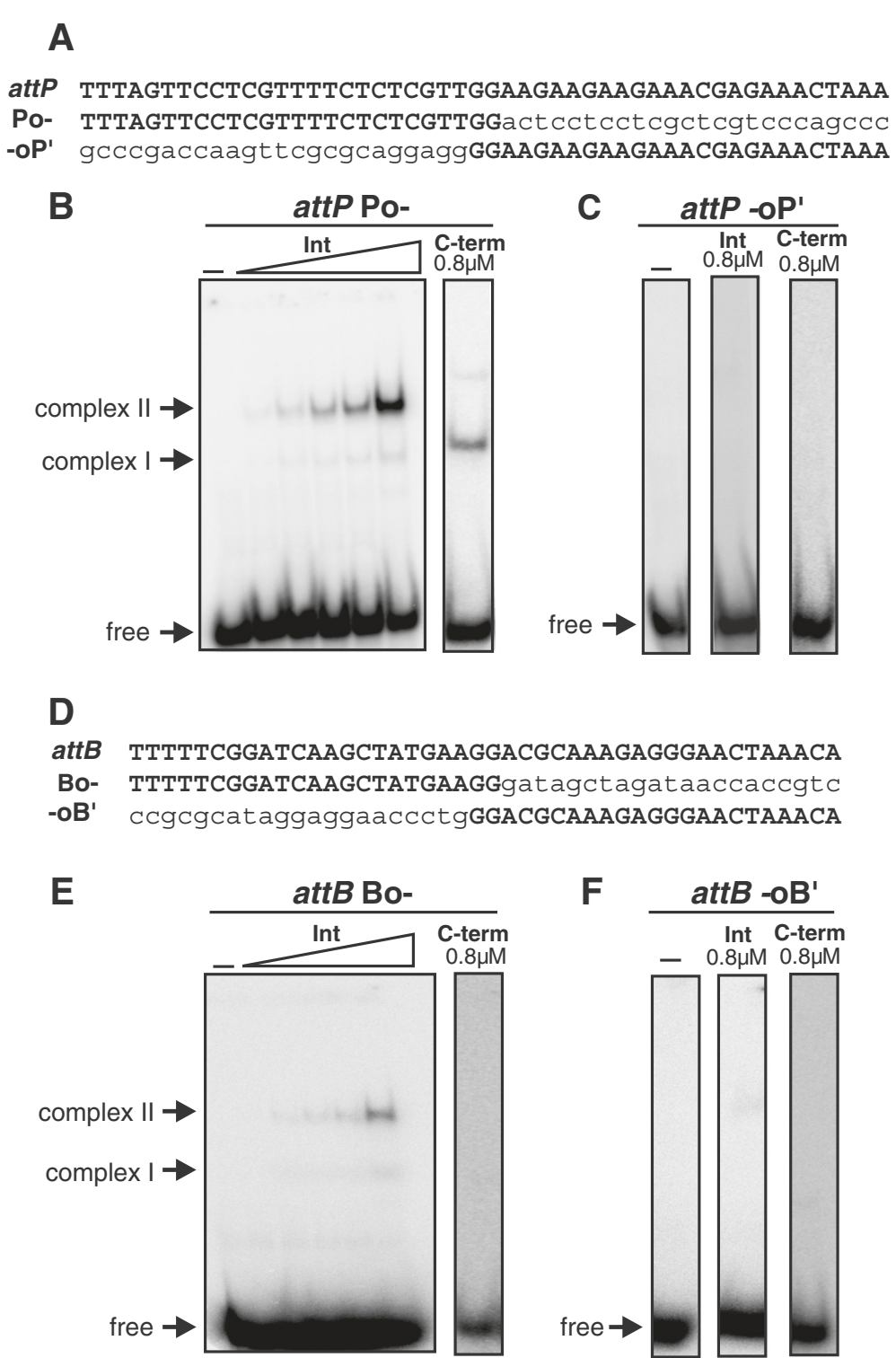

Figure 6 Integrase binding to half-att sites. (A) Sequences of attP half-sites (Po- and -oP'). Lowercase letters designate non-att sequences on the duplex probes. (B) Gel mobility shift assay with the Po- half-site. Left panel contains increasing concentrations of integrase $(0,10,30,90,270$, and $810 \mathrm{nM}$ ), and right panel shows binding with $810 \mathrm{nM}$ of the C-terminal integrase domain. (C) Gel mobility shift assay with the -oP' half-site using $810 \mathrm{nM}$ of integrase or the C-terminal domain. (D) Sequences of attB half-sites (Bo- and -oB'). (E) Gel mobility shift assay with the Bo- halfsite. Left panel contains increasing concentrations of integrase $(0,10,30,90,270$ and $810 \mathrm{nM})$, and right panel shows binding with $810 \mathrm{nM}$ of the C-terminal domain. (F) Gel mobility shift assay with the -oB' half-site using $810 \mathrm{nM}$ of integrase and the C-terminal domain.

symmetrical such that both standard parallel (Figure $8 \mathrm{~F}$ ) as well as antiparallel (Figure $8 \mathrm{G}$ ) synapses may be productive for recombination. When the products of in vitro recombination reactions were analyzed in the double mutants using restriction enzymes whose cleavage distinguishes between inversion and deletion, both product types were present. $\operatorname{attP}(\mathrm{G} 1 \mathrm{C}) \times \operatorname{attB}(\mathrm{G} 1 \mathrm{C})$ generated $64.5 \%$ deletion and $35.5 \%$ inversion products, and attP $(\mathrm{G} 1 \mathrm{C}) \times a t t B(\mathrm{G} 1 \mathrm{C})$ generated $52.5 \%$ deletion and $47.5 \%$ inversion products. Sequence analysis of representative plasmids obtained in the in vivo experiment in (E) confirmed that the white $\left(l a c Z^{-}\right)$plasmids were the predicted site-specific deletion products and that the blue plasmids contained inversions with hybrid $a t t L$ and $a t t R$ sites as denoted in (G). Recombination sites containing a symmetric dinucleotide core sequence can thus productively synapse in either orientation to generate both deletion and inversion products. The native asymmetric GG dinucleotides must then solely specify directionality of the att sites in the A118 integrase system. 

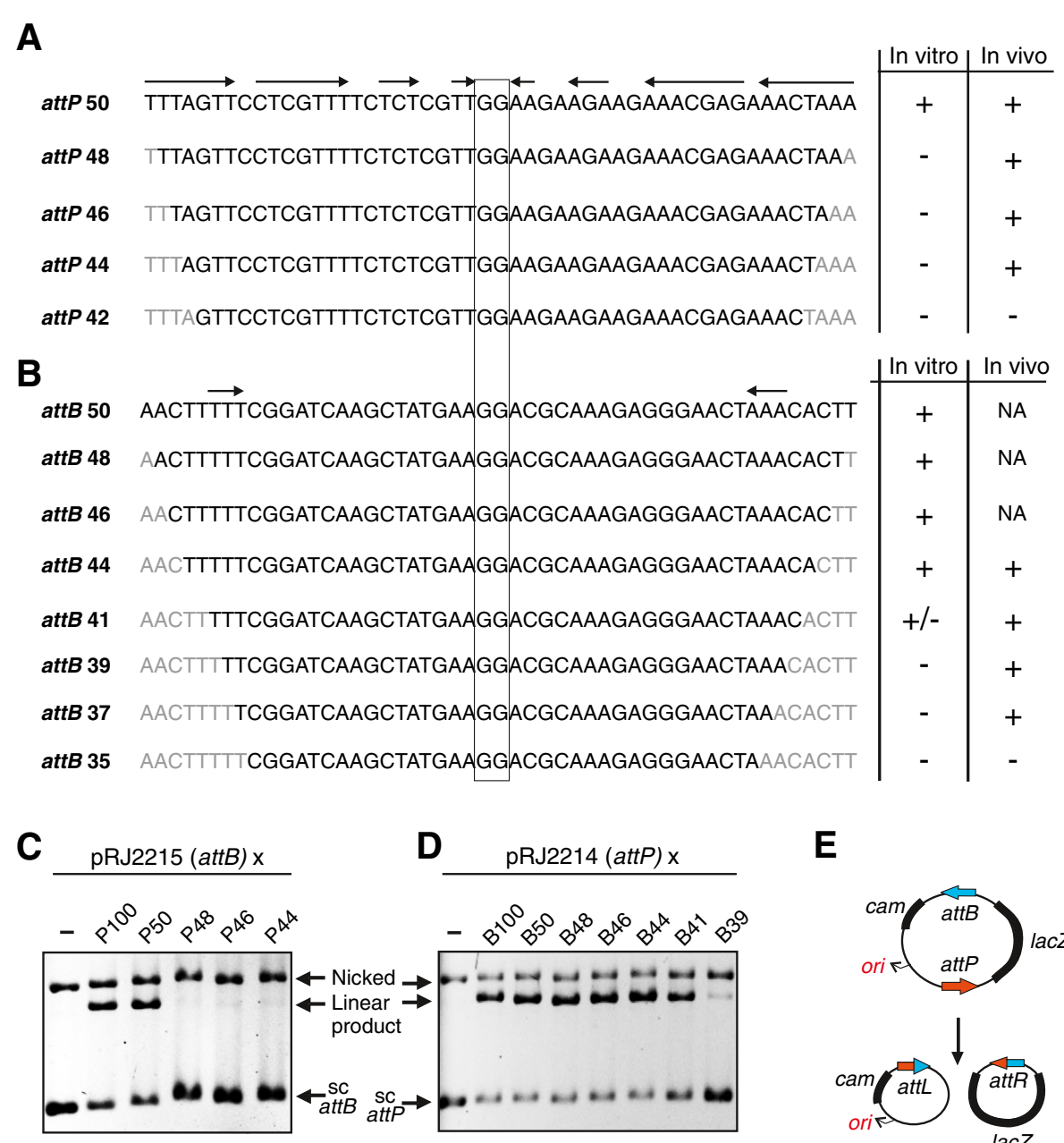

$\mathbf{E}$

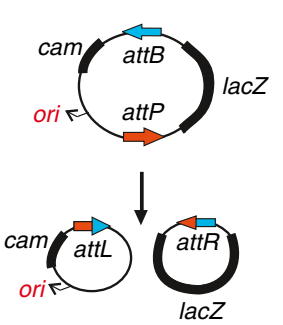

Figure 7 Determination of minimal attB and attP lengths required for efficient recombination. (A) Sequence of the resected attP substrates and their abilities to support in vitro intermolecular integration (for example (C)) or in vivo intramolecular deletion (for example (E)). Symmetry-related segments denoted above the attP and attB sequences, and the GG dinucleotide crossover segments (see Figure 8) are boxed. In vitro data: (+), >60\% recombinants; (-), little-no detectable products in 40-minute reactions. In vivo data: (+), >90\% white colonies (deletion products); $(-),<10 \%$ white colonies. Sequences in light grey are absent in the in vitro reactions and replaced with different nucleotides in the in vivo reactions. (B) Sequence of the resected attB substrates and their abilities to support in vitro intermolecular integration (for example (D)) or in vivo intramolecular deletion. Activities are designated as in (A); in vitro reaction rates for attB 41 (+/-) were about $15 \%$ of those of the longer substrates. NA, not analyzed. (C), (D) Representative gels showing in vitro intermolecular integration reactions (40 minutes) between supercoiled attB (pRJ2215) and resected attP fragments (C) or between supercoiled attP (pRJ2214) and resected attB fragments. (E) Illustration of the in vivo attPxattB deletion reaction with pBCPB-A1+ [31]. Deletion generates two circular products that may be topologically linked, but only the lacZ $\mathrm{Cam}^{r}$ product containing the replication origin will be maintained.

\section{Discussion}

In this work we have established and characterized a robust in vitro attP $\times$ attB integration reaction by the A118 integrase. We have used this system to elucidate basic properties of the A118 integrase including its domain structure and binding properties to the recombination sites. We have also defined the DNA sequence boundaries specifying the attP and attB recombination sites and mapped the $2 \mathrm{bp}$ region where DNA exchange occurs. The sequence of these two core base pairs defines the orientation of the att sites. Thus far we find that the A118 integration reaction shares fundamental properties with other characterized reactions by members of the serine recombinase, especially those of the integrase subfamily containing large $\mathrm{C}$-terminal domains.

Partial proteolysis of the A118 integrase by chymotrypsin or proteinase $\mathrm{K}$ demonstrated that the full-length enzyme consists of two distinct folded domains, an $\mathrm{N}$ terminal domain corresponding to the catalytic core that is common to all serine recombinases linked to a large domain that constitutes over two-thirds of the protein (Figure 2E). The large C-terminal domain mediates att site binding as is observed for other members of this subfamily $[24,29,42]$. Given the substrate specificity of chymotrypsin, 

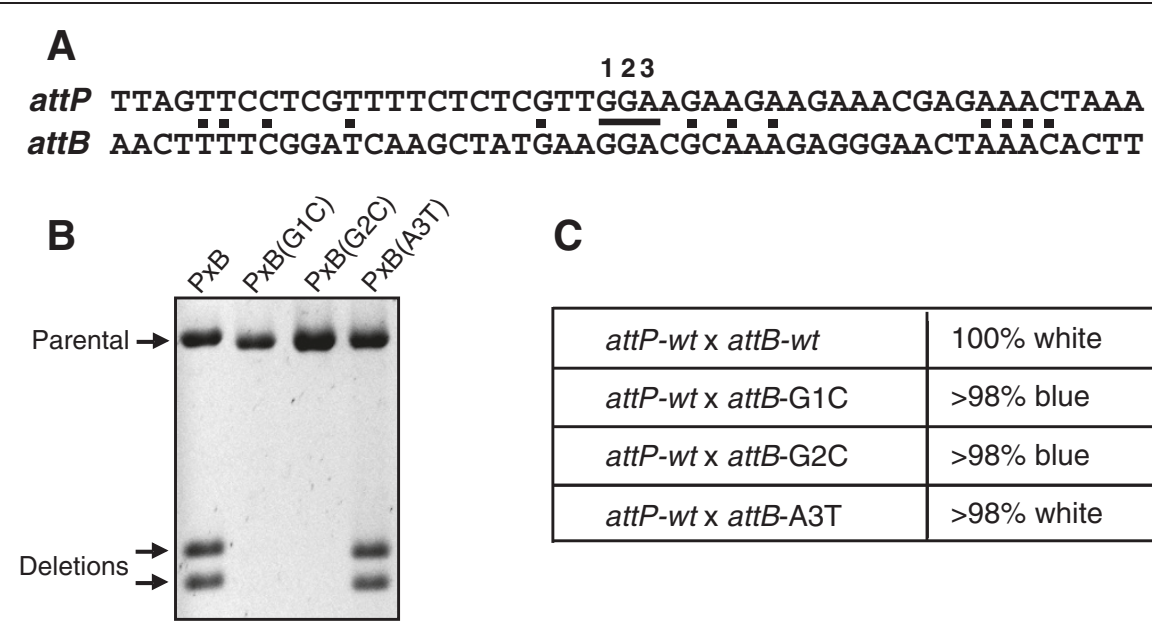

C

\begin{tabular}{|l|l|}
\hline attP-wt $\times$ attB-wt & $100 \%$ white \\
\hline attP-wt $\times$ attB-G1C & $>98 \%$ blue \\
\hline attP-wt $\times$ attB-G2C & $>98 \%$ blue \\
\hline attP-wt $\times$ attB-A3T & $>98 \%$ white \\
\hline
\end{tabular}

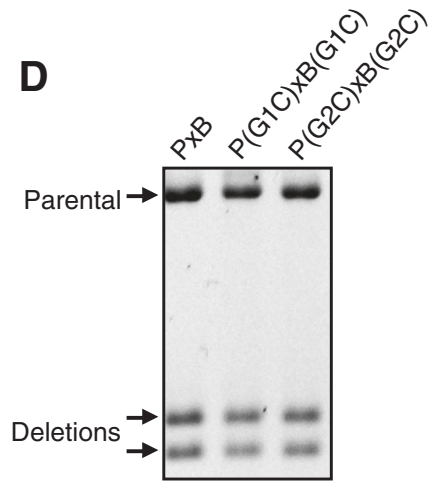

$\mathbf{E}$

\begin{tabular}{|l|l|}
\hline attP-wt $\mathrm{x}$ attB-wt & $100 \%$ white \\
\hline attP-G1C $\times$ attB-G1C & $\begin{array}{l}55 \% \text { blue } \\
45 \% \text { white }\end{array}$ \\
\hline attP-G2C $\times$ attB-G2C & $\begin{array}{l}45 \% \text { blue } \\
55 \% \text { white }\end{array}$ \\
\hline attP-A3T $\mathrm{x}$ attB-A3T & $>98 \%$ white \\
\hline
\end{tabular}

$\mathbf{F}$

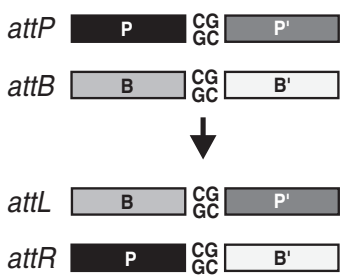

G

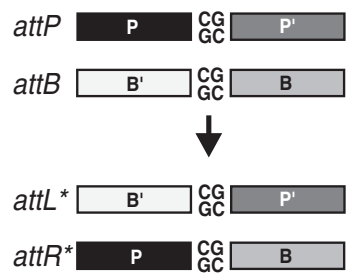

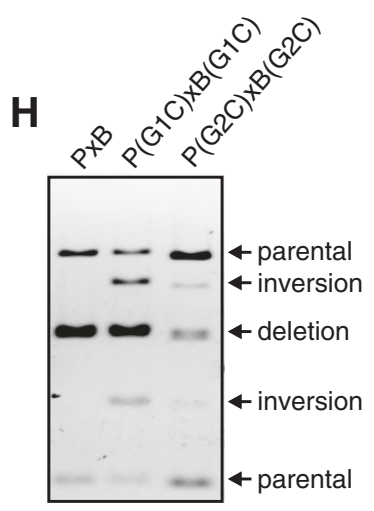

Figure 8 Determination of the crossover site. (A) Sequence of the minimal attP and attB sites, with common nucleotides marked with dots. The central three nucleotides are numbered 1 to 3 in this figure. (B) In vitro intramolecular deletion reactions (30 minutes) on supercoiled plasmids with $a t t B$ sites containing mutations at the central three nucleotides as designated. $P \times B$, wild-type control. The products were digested with $\mathrm{Xhol}$ and $\mathrm{BamH1}$ to reveal deletion products (Figure 1D). (C) Results of in vivo deletion reactions between attP and mutated attB sites. White colonies result from site-specific deletions and blue colonies signify absence of deletions. (D) In vitro intramolecular recombination reactions (30 minutes) with plasmid substrates containing identical changes within attP and attB at the designated positions. Parental and deletion product bands are denoted. (E) Results of in vivo recombination reactions between mutated attP and attB sites. Because G1C and G2C mutations create symmetrical cores, inversions between att sites oriented in an antiparallel configuration (G) form along with deletions. Inversion products retain the $\mathrm{Lac}^{+}$(blue) phenotype. (F) Diagram of synapsis between attP(G1C) and attB(G1C) in the standard parallel orientation generating attL and attR upon DNA exchange. Productive recombination between wild-type attP and attB sites containing the asymmetric GG core nucleotides only occur by this pathway. (G) Diagram of an antiparallel synapsis between $\operatorname{attP(G1C)}$ and $\operatorname{attB}(G 1 C)$ generating the hybrid attL $L^{*}$ and attR* sites upon DNA exchange. (H) In vitro intramolecular recombination reactions (30 minutes) with plasmid substrates containing identical changes within attP and attB within the core nucleotides. The reaction products were digested with BamHI, Ndel, and Scal to reveal both deletions (doublet bands) and inversions as denoted. 
the site for chymotrypsin cleavage is most likely to be at Met136. When compared with available X-ray structures, the protease sensitive site would be within the oligomerization $\alpha$-helix E segment, shortly past the region that is predicted to be in contact with the catalytic core. A118 integrase Met136 corresponds to $\gamma \delta$ resolvase residue 126 that is located in the region of helix $\mathrm{E}$ that becomes disordered in the absence of DNA [43]. When bound to DNA, integrase becomes largely resistant to chymotrypsin, consistent with formation of an extended helix, as seen for the $\gamma \delta$ resolvase dimer-DNA complex [44]. The segment within the predicted helix E region of integrase, however, remains sensitive to proteinase $\mathrm{K}$ activity in the presence of attP DNA.

Proteinase $\mathrm{K}$ digestion generates an additional fragment that corresponds to cleavages at the C-terminal end of the catalytic core (at or prior to residue 96 corresponding to near the C-terminal end of a putative helix D) and shortly past the basic cysteine/histidine-rich segment within the C-terminal domain (at or shortly beyond residue 331 at the end of a predicted $\alpha$-helix). Significantly, this fragment is not observed when integrase is bound to attP. Potential models - in light of the fact that the $\mathrm{C}$-terminal domain mediates DNA binding - are that bound attP DNA directly occludes the protease or induces a conformation change that masks the scission site near residue 331 . These would result in the accumulation of a stable $\mathrm{C}$-terminal proteinase $\mathrm{K}$ product from approximately residue 136 to the C-terminal end as observed in Figure 2D (lanes 7 and 8). The analogous cysteine-rich segment, together with zinc, has been implicated to be involved in binding DNA by the $\phi C 31$ integrase [32]. Our results are consistent with this region being involved directly or indirectly in DNA binding, but we have not obtained evidence for a role of zinc in DNA binding or recombination by A118 integrase. Most other serine integrases also are reported to function effectively without zinc [27,33-35]. The precise mode of DNA binding remains to be determined for any of the large serine recombinase subfamily members, and data accumulated from several systems suggest there may be significant differences [23].

We found that full-length A118 integrase binds att DNA in a highly cooperative manner consistent with it being a dimer in solution. The dimer is even the dominant form bound to half-att sites, although binding is poor. On the contrary, the isolated C-terminal domain, which chromatographs as a monomer and exhibits no evidence for multimerization in solution by amine crosslinking (data not shown), binds noncooperatively to attP or $a t t B$. This is different to that observed for the $C$ terminal domain of $\phi C 31$ integrase, which cooperatively binds to its respective att sites even though it is also monomeric in solution [28]. Dimerization by the intact protein appears extremely sensitive to the zwitterionic detergent CHAPS, even more sensitive than the Hin invertase. The effect of CHAPS on att binding, particularly at $a t t B$ where both half-sites exhibit low affinity, is mirrored by its inhibitory effect on recombination. The sensitivity to CHAPS, together with the modest stimulation by ethylene glycol and glycerol, suggests that fundamental features of the dimer to tetramer transition involving the oligomerization helix that presumably accompany formation of integrase synaptic complexes are likely to share similarities with Hin $[10,40,41,45]$.

The A118 integrase only efficiently supports recombination between $a t t P$ and $a t t B$, and this reaction is relatively efficient regardless of whether the sites are located on separate DNA molecules or in cis (either in an inverted or directly repeated orientation). Surprisingly, a low efficiency reaction between two attP sites was observed in vitro, but importantly with respect to the phage lifecycle no reactions involving attL or attR sites were detected with integrase alone. Binding of integrase to each of the four att sites is similar and thus cannot explain the site selectivity for recombination. We have recently identified an additional phage-encoded protein that is required for the excisive $a t t L \times a t t R$ reaction, which will be the subject of a future report. Other serine integrases also exhibit exquisite site-selectivity for recombination [24,26,27,33] and have been found to require recombination directionality factors or Xis-like proteins for attL $\times a t t R$ recombination [27,46-48]. Mechanisms underlying site-selective synapsis are a major area of exploration for the integrase subfamily of serine recombinases.

As is the case for most of the other serine integrases [23], the sequences defining the A118 attP and attB sites are remarkably unrelated (Figure 7 ). Unlike most of the other characterized systems, the minimal A118 attB site exhibits essentially no symmetry. The A118 attP site requires specific sequences extending 22 bp on either side of the 2 bp crossover site, and $a t t B$ requires about $19 \mathrm{bp}$. An additional $3 \mathrm{bp}$ of flanking DNA is required for minimal att function, presumably reflecting DNA backbone contacts needed for A118 integrase binding. Whereas minimal att site lengths vary somewhat between systems, most require between 35 and $55 \mathrm{bp}$. Similar to A118, many integrases such as Bxb1, $\phi C 31$, $\phi$ BT1, $\phi$ Rv1, TG1, and TP901 [24,27,33,34,49,50], but not all (for example, R4 [51]), require a longer sequence at attP. Both full-length and the isolated C-terminal domain of $\mathrm{A} 118$ integrase interact with $a t t P$ and $a t t B$ asymmetrically, with the attP and $a t t B$ left half-sites exhibiting much higher affinity than the right half-sites. Whether this difference is functionally important is not known, but a symmetrical $a t t B(\mathrm{BoB})$ containing two good half-sites was a poor recombination substrate (data not shown). The sequence asymmetry cannot be a 
determinant of orientation-dependent att site synapsis since sites with symmetric core base pairs efficiently recombined from both parallel and antiparallel synapses (Figure 8E,F,G,H). The $\phi C 31$ attB and Bxb1 attP sites have also been reported to bind to their respective integrases in an asymmetric manner [24,52].

Finally we note that the in vitro A118 attP $\times a t t B$ reaction is efficient under a variety of substrate topology and solution conditions. The most efficient reaction occurs when the att sites are located in cis on a supercoiled molecule in the presence of spermidine and divalent cations where rates up to three recombinants per minute are obtained. However, the reaction occurs readily in the presence of metal chelators and without a polyamine and on linear DNA. These properties are generally similar to other serine integrases but contrast with members of the resolvase/invertase subfamily $[17,18]$. A mechanistically interesting feature of the A118 integrase deletion reaction is the formation of topologically unlinked circular deletion products, even when the att sites are separated by many kilobases on a supercoiled DNA molecule (unpublished data). The abundance of free deletion products implies constraints on the assembly of the synaptic complex and the subunit rotation reaction.

\section{Conclusions}

There are a number of fundamental questions regarding the mechanism of recombination reactions catalyzed by serine integrases, particularly with respect to the roles of their C-terminal domains, whose sizes dwarf the much smaller catalytic domains. These include, but are not limited to, mechanisms underlying the exquisite site selectivity for productive synapsis, and the architecture of the active recombination complex. In this report we have characterized the biochemical properties and substrate requirements of the $a t t P \times a t t B$ integration reaction by the phage A118 integrase. The reaction is robust and amenable to more detailed studies on its reaction mechanism. The A118 recombinase thus joins the handful of model serine integrase systems where in vitro studies are advancing our understanding of this subfamily. Studies on members from the different subclasses (Figure 1B) will almost certainly reveal both unique and common features of the subfamily. Information from the different systems will be essential for a full understanding of how these enzymes function and how they can be utilized to their fullest potential for genetic engineering.

\section{Methods}

\section{Strains, phages and recombinant plasmids}

A list of plasmids used in this work is presented in Table 1. Richard Calendar (University of California - Berkeley) kindly provided the following $L$. monocytogenes strains and DNA: DP-L3689 (strain 10403 lysogenized with
A118), DP-L3670 (strain 10403S lysogenized with U153), DP-L4056 (strain 10403S cured of phage), and phages A118 and U153. The A118 integrase coding sequence was amplified by PCR from the phage DNA and cloned between NdeI and BamHI sites of pET11a and pET15b (EMD Millipore Billerica, MA USA) to give pRJ2186 and pRJ2184, respectively. pRJ2823 contains integrase residues 158 to 452 (C-terminus) in pET15b. The att sites were initially cloned into pBR322 with 100 or $200 \mathrm{bp}$ of native sequence flanking on each side as PCR fragments using DNA from the phage, lysogen, or cured strain.

Substrates for in vivo recombination assays were derived from pBCPB-A1+, which was provided by Michele Calos (Stanford University, CA, USA). pBCPB-A1+ is a colE1based plasmid that contains the lac $Z$ gene flanked in direct repeat orientation with $200 \mathrm{bp} a t t B$ (between $\mathrm{BamHI}$ and XhoI sites) and attP (SmaI site) segments [31]. A deletion reaction results in 3.0 plus $3.4 \mathrm{~kb}$ product circles (Figure 3D). pRJ2799 contains the $a t t P$ and $a t t B$ sites in inverted orientation relative to each other and was constructed by substituting 200 bp A118 attP and attB sites into SmaI and BamHI-XhoI sites, respectively, in $\mathrm{pBCPB}+$ [51]. pRJ2913 contains attR and attL in direct repeat orientation and was constructed in a similar manner as described for pRJ2799. Integrase for in vivo assays was supplied by pInt that has the A118 integrase controlled by lacP on a p15A origin plasmid [31]. For minimal att site length determination, different length $a t t P$ or $a t t B$ duplex oligonucleotides were substituted for their respective sites in $\mathrm{pBCPB}-\mathrm{A} 1+$. Individual base pair substitutions within att sites were generated by QuikChange mutagenesis.

\section{Integrase purification}

RJ3386 (BL21(DE3) endA::Tn10) containing His-tagged integrase overexpressing plasmids were grown in $1 \mathrm{l} \mathrm{LB}$ with ampicillin $(100 \mu \mathrm{g} / \mathrm{ml})$ at $37^{\circ} \mathrm{C}$ until the optical density at $600 \mathrm{~nm}$ reached 0.6 . The cultures were cooled to $10^{\circ} \mathrm{C}$, isopropylthio- $\beta$-galactoside added to $0.5 \mathrm{mM}$, and incubation continued for 18 to 20 hours at $10^{\circ} \mathrm{C}$ with shaking. Harvested cells were resuspended in $20 \mathrm{ml}$ lysis buffer (20 mM HEPES, pH 7.5, $200 \mathrm{mM} \mathrm{NaCl}$, $20 \mathrm{mM}$ imidazole, $1 \mathrm{mM}$ DTT, $10 \%$ glycerol, and 0.1 $\mathrm{mM}$ phenylmethanesulfonyl fluoride and lysed by two passes through a French press. The lysate was centrifuged at $26,000 \times g$ for 30 minutes, and the supernatant loaded onto a $1 \mathrm{ml} \mathrm{Ni-NTA}$ column that was then washed with $20 \mathrm{mM}$ HEPES, pH 7.5, $0.5 \mathrm{M} \mathrm{NaCl}$, $40 \mathrm{mM}$ imidazole and 10\% glycerol, and bound protein eluted with the same buffer but with $0.3 \mathrm{M} \mathrm{NaCl}$ and 200 mM imidazole. Full-length ${ }^{\mathrm{His}}$ Int was then loaded directly onto a column containing $0.5 \mathrm{ml}$ Heparin Sepharose 6 Fast Flow (GE Healthcare Biosciences, Pittsburgh, PA USA) column, washed with $20 \mathrm{mM}$ HEPES, pH 7.5, 0.5 M $\mathrm{NaCl}, 1 \mathrm{mM}$ DTT, and 10\% glycerol, and eluted with the 
Table 1 Plasmids used in the present study

\begin{tabular}{|c|c|c|}
\hline Plasmid & Description $^{a}$ & Source \\
\hline pRJ2184 & pET15b, A118 integrase between Ndel-BamH1 & This work \\
\hline pRJ2186 & pET11a, A118 integrase between Ndel-BamH1 & This work \\
\hline pRJ2823 & pET15b, A118 integrase ${ }^{158-452}$ between Ndel-BamH1 & This work \\
\hline $\mathrm{pRJ} 2214$ & pBR322, attP (400 bp) into EcoR1 & This work \\
\hline $\mathrm{pRJ} 2215$ & pBR322, attB (200 bp) into Sall & This work \\
\hline pRJ2289 & pBR322, attP U153 (200 bp) into EcoR1 & This work \\
\hline $\mathrm{pBCPB}+$ & pBCSK+, cam (lacZ) & Groth and colleagues [50] \\
\hline plntA1 & pACYC177, kan lacP-A118 integrase & Keravala and colleagues [31] \\
\hline pBCPB-A1+ & pBCSK+, cam DR(attB-lacZ-attP) & Keravala and colleagues [31] \\
\hline pRJ2799 & pBCPB+, cam IR(attB-lacz-attP) & This work \\
\hline pRJ2825 & $\mathrm{pBCPB}+, \operatorname{cam} \mathrm{DR}(\mathrm{attB}(\mathrm{G} 1 \mathrm{C})-\mathrm{lac} Z$-attP) & This work \\
\hline pRJ2826 & pRJ2825, attP (G1C) & This work \\
\hline pRJ2827 & $\mathrm{pBCPB}+, \operatorname{cam} \mathrm{DR}(\mathrm{attB}(\mathrm{G} 2 \mathrm{C})-\mathrm{lac} Z$-attP) & This work \\
\hline pRJ2828 & pRJ2827, attP (G2C) & This work \\
\hline pRJ2829 & pBCPB+, cam DR(attB(A3T)-lacZ-attP) & This work \\
\hline pRJ2830 & pRJ2829, attP (A3T) & This work \\
\hline pRJ2831 & $\mathrm{pBCPB}+$, cam DR(attB ${ }^{44}$-lacZ-attP) & This work \\
\hline pRJ2927 & $\mathrm{pBCPB}+$, cam DR(attB ${ }^{41}$-lacZ-attP) & This work \\
\hline pRJ2832 & pBCPB+, cam DR(attB ${ }^{39}$-lacZ-attP) & This work \\
\hline $\mathrm{pRJ} 2833$ & $\mathrm{pBCPB}+$, cam DR(attB ${ }^{37}$-lacZ-attP) & This work \\
\hline pRJ2834 & $\mathrm{pBCPB}+$, cam DR(attB ${ }^{35}$-lacZ-attP) & This work \\
\hline pRJ2835 & $\mathrm{pBCPB}+$, cam DR $\left(a t t B-l a c Z-a t t P^{50}\right)$ & This work \\
\hline pRJ2836 & $\mathrm{pBCPB}+$, cam DR(attB-lacZ-attP $\left.{ }^{48}\right)$ & This work \\
\hline pRJ2848 & pBCPB+, cam DR(attB-lacZ-attP46) & This work \\
\hline pRJ2837 & $\mathrm{pBCPB}+$, cam DR(attB-lacZ-attP $\left.{ }^{44}\right)$ & This work \\
\hline pRJ2928 & $\mathrm{pBCPB}+$, cam DR(attB-lacZ-attP $\left.{ }^{42}\right)$ & This work \\
\hline $\mathrm{pRJ} 2913$ & pBCPB+, cam DR(attL-lacZ-attR) & This work \\
\hline \multicolumn{3}{|l|}{ Strain } \\
\hline \multicolumn{2}{|c|}{ DP-L 3689 (10403::A118) } & R Calendar \\
\hline \multicolumn{2}{|c|}{ DP-L 3670 (10403S::U153) } & R Calendar \\
\hline \multicolumn{2}{|c|}{ DP-L 4056 (10403S phage cured) } & R Calendar \\
\hline
\end{tabular}

att sites are from A118 unless specified. DR, att sites in direct orientation; IR, att sites in inverted orientation.

same with $1 \mathrm{M} \mathrm{NaCl}$. Native integrase was obtained by heparin-Sepharose chromatography essentially as above. Integrase preparations were stored in $20 \mathrm{mM}$ HEPES, $\mathrm{pH}$ 7.5, $1 \mathrm{M} \mathrm{NaCl}, 1 \mathrm{mM}$ DTT, $0.1 \mathrm{mM}$ EDTA and 40\% glycerol at $-20^{\circ} \mathrm{C}$.

Size exclusion chromatography was performed through an FPLC Superdex-200 10/300 GL column (GE Healthcare Biosciences, Pittsburgh, PA USA) in 20 mM HEPES, $\mathrm{pH} 7.5,1 \mathrm{M} \mathrm{NaCl}, 1 \mathrm{mM}$ DTT, and $10 \%$ glycerol at a flow rate of $0.4 \mathrm{ml} / \mathrm{minute}$ at $23^{\circ} \mathrm{C}$.

\section{In vitro recombination reactions}

Standard recombination reactions were typically performed in a buffer containing $20 \mathrm{mM}$ HEPES, pH 7.5, $100 \mathrm{mM} \mathrm{NaCl}$ or $\mathrm{KCl}, 5 \mathrm{mM}$ spermidine, $2.5 \mathrm{mM}$ DTT, $5 \mathrm{mM} \mathrm{CaCl}_{2}$ or $\mathrm{MgCl}_{2}, 30 \mu \mathrm{g} / \mathrm{ml} \mathrm{BSA}$, and $5 \%$ glycerol and incubation was at $30^{\circ} \mathrm{C}$. Intramolecular deletion and inversion reactions were performed with 0.02 pmol plasmid DNA and intermolecular integration reactions typically utilized 0.03 pmol supercoiled plasmid DNA and 0.09 pmol linear DNA (50 to 100 bp fragment derived from PCR or synthetic duplex oligonucleotides). Reactions were initiated by addition of 0.5 to 1 pmol purified integrase and terminated by inactivation at $65^{\circ} \mathrm{C}$ or addition of $1 \%$ SDS. Intramolecular recombination reactions were digested with $\mathrm{BamHI}$ and $\mathrm{XhoI}$ restriction enzymes to generate linear deletion products or reveal inversion prior to agarose gel electrophoresis.

\section{In vitro DNA binding assays}

DNA probes were prepared by polynucleotide kinase reactions with $\gamma^{-}{ }^{32} \mathrm{P}$-ATP of gel-purified PCR-generated 
fragments or oligonucleotides. Varying concentrations of integrase were added to approximately $0.1 \mathrm{pmol}{ }^{32} \mathrm{P}$ DNA in a buffer containing $20 \mathrm{mM}$ HEPES, pH 7.5, 100 $\mathrm{mM} \mathrm{NaCl}, 0.1 \mathrm{mg} / \mathrm{ml} \mathrm{BSA}, 5 \mathrm{mM}$ spermidine, $5 \mathrm{mM}$ DTT, $5 \%$ glycerol, and $50 \mu \mathrm{g} / \mathrm{ml}$ sonicated salmon sperm DNA in a total volume of $10 \mu \mathrm{l}$. The reactions were incubated at $30^{\circ} \mathrm{C}$ for 30 minutes and the protein-DNA complexes were separated on nondenaturing $6 \%$ polyacrylamide gels in $0.5 \times \mathrm{TBE}$ buffer at room temperature. Gels were dried and analyzed by phosphorimaging.

\section{In vivo recombination assays}

Recombination substrate plasmids $\left(\mathrm{Cam}^{\mathrm{r}}\right)$ were transformed into E. coli DH5 $\alpha$ containing pIntA1 $\left(\mathrm{Kan}^{\mathrm{r}}\right)$ and plated on LB agar plates containing chloramphenicol, kanamycin, and X-gal $(20 \mu \mathrm{g} / \mathrm{ml})$. After overnight incubation at $37^{\circ} \mathrm{C}$, blue (unrecombined) and white (deletion) colonies were scored.

\section{Partial proteolysis and mass spectrometry}

Different amounts of chymotrypsin (Sigma-Aldrich, St. Louis, MO USA) and proteinase K (Sigma-Aldrich) were added to $3 \mu \mathrm{g}$ integrase in $20 \mu \mathrm{l}$ PBS and incubated at $23^{\circ} \mathrm{C}$ for 10 minutes. 4-(2-Aminoethyl) benzenesulfonyl fluoride hydrochloride $(2.5 \mathrm{mg} / \mathrm{ml})$ and phenylmethanesulfonyl fluoride $(2 \mathrm{mM})$ were added to terminate the chymotrypsin and proteinase $\mathrm{K}$ reactions, respectively. SDS sample buffer was then added, the samples were heated at $95^{\circ} \mathrm{C}$ for 10 minutes, and proteolysis products were analyzed by $15 \%$ SDS-PAGE. For partial proteolysis of integrase in the presence of DNA, integrase was incubated with a twofold molar excess of attP (50 bp) oligonucleotides at $23^{\circ} \mathrm{C}$ for 10 minutes before digestion. For mass spectrometry, gel fragments were excised, in-gel digested with trypsin (Trypsin Gold, Mass Spectrometry Grade; Promega Madison, WI USA), and analyzed using positive reflector mode on a MALDI-TOF/TOF Ultraflex instrument (Bruker Daltonics Inc., Billerica, MA, USA). Peptides were identified using the Mascot Server software v.2.2 (Matrix Science Ltd., London, UK).

\section{Abbreviations \\ Bp: Base pair; BSA: Bovine serum albumin; CHAPS: 3-((3-cholamidopropyl) dimethylammonio)-1-propanesulfonate; DTT: Dithiothreitol; \\ EDTA: Ethylenediamine tetraacetic acid; $\mathrm{K}_{\mathrm{d}}$ : Dissociation constant; LB: Luria Bertani broth; MALDI-TOF/TOF: Matrix-assisted laser desorption/ionization time of flight/time of flight; PBS: Phosphate-buffered saline; PCR: Polymerase chain reaction; SSR: Site-specific recombination.}

\section{Competing interests}

The authors declare that they have no competing interests.

\section{Authors' contributions}

SM generated most of the data in the manuscript. GD initiated work on the project and generated early data. NKA and MJH performed the mass spectrometry. SM, GD, and RCJ participated in the design of the study. RCJ and SM drafted the manuscript. All authors read and approved the final manuscript.
Authors' information

Joint first author: Sridhar Mandali and Gautam Dhar.

\section{Acknowledgements}

The authors thank Richard Calendar (University of California - Berkeley) and Michele Calos (Stanford University) for providing strains and plasmids used in this work, and lan Barr for help with the size exclusion chromatography. This work was supported by NIH grant GM038509.

\section{Author details}

'Department of Biological Chemistry, David Geffen School of Medicine at UCLA, Los Angeles, CA 90095-1737, USA. ${ }^{2}$ Present address: Department of Obstetrics and Gynecology, David Geffen School of Medicine at UCLA, Los Angeles, CA 90095, USA. ${ }^{3}$ Molecular Biology Institute, University of California, Los Angeles, Los Angeles, CA 90095, USA.

Received: 24 July 2012 Accepted: 25 October 2012

Published: 3 January 2013

\section{References}

1. Loessner MJ, Inman RB, Lauer P, Calendar R: Complete nucleotide sequence, molecular analysis and genome structure of bacteriophage A118 of Listeria monocytogenes: implications for phage evolution. Mol Microbiol 2000, 35:324-340.

2. Craig NL, Craigie R, Gellert M, Lambowitz AM: Mobile DNA II. Washington, DC: ASM Press; 2002.

3. Groth AC, Calos MP: Phage integrases: biology and applications. J Mol Biol 2004, 335:667-678

4. Sauer B: Site-specific recombination: developments and applications. Curr Opin Biotechnol 1994, 5:521-527.

5. Bonnet J, Subsoontorn P, Endy D: Rewritable digital data storage in live cells via engineered control of recombination directionality. Proc Natl Acad Sci U S A 2012, 109:8884-8889.

6. Branda CS, Dymecki SM: Talking about a revolution: the impact of sitespecific recombinases on genetic analyses in mice. Dev Cell 2004, 6:7-28.

7. Grindley ND, Whiteson KL, Rice PA: Mechanisms of site-specific recombination. Annu Rev Biochem 2006, 75:567-605.

8. Van Duyne GD: A structural view of cre-loxP site-specific recombination. Annu Rev Biophys Biomol Struct 2001, 30:87-104.

9. Chen Y, Rice PA: New insight into site-specific recombination from Flp recombinase-DNA structures. Annu Rev Biophys Biomol Struct 2003, 32:135-159.

10. Dhar G, Heiss JK, Johnson RC: Mechanical constraints on Hin subunit rotation imposed by the Fis/enhancer system and DNA supercoiling during site-specific recombination. Mol Cell 2009, 34:746-759.

11. Li W, Kamtekar S, Xiong Y, Sarkis GJ, Grindley ND, Steitz TA: Structure of a synaptic gamma delta resolvase tetramer covalently linked to two cleaved DNAs. Science 2005, 309:1210-1215.

12. Dhar G, Sanders ER, Johnson RC: Architecture of the Hin synaptic complex during recombination: the recombinase subunits translocate with the DNA strands. Cell 2004, 119:33-45.

13. Stark WM, Sherratt DJ, Boocock MR: Site-specific recombination by Tn3 resolvase: topological changes in the forward and reverse reactions. Cell 1989, 58:779-790.

14. Johnson RC, McLean MM: Recombining DNA by protein swivels. Structure 2011, 19:751-753.

15. Keenholtz RA, Rowland SJ, Boocock MR, Stark WM, Rice PA: Structural basis for catalytic activation of a serine recombinase. Structure 2011, 19:799-809.

16. Smith M, Thorpe H: Diversity in the serine recombinases. Mol Micro 2002, 44:299-307.

17. Grindley NDF: The movement of Tn3-like elements: Transposition and cointegrate resolution. In Mobile DNA II. Edited by Craig NL, Craigie R, Gellert M, Lambowitz AM. Washington, DC: ASM Press; 2002:272-302.

18. Johnson RC: Bacterial site-specific DNA inversion systems. In Mobile DNA II. Edited by Craig NL, Craigie R, Gellert M, Lambowitz AM. Washington, DC ASM Press; 2002:230-271.

19. Dhar G, McLean MM, Heiss JK, Johnson RC: The Hin recombinase assembles a tetrameric protein swivel that exchanges DNA strands. Nucleic Acids Res 2009, 37:4743-4756.

20. Mouw KW, Rowland SJ, Gajjar MM, Boocock MR, Stark WM, Rice PA: Architecture of a serine recombinase-DNA regulatory complex. Mol Cell 2008, 30:145-155. 
21. Nollmann M, He J, Byron O, Stark WM: Solution structure of the Tn3 resolvase-crossover site synaptic complex. Mol Cell 2004, 16:127-137.

22. Yuan P, Gupta K, Van Duyne GD: Tetrameric structure of a serine integrase catalytic domain. Structure 2008, 16:1275-1286.

23. Smith MC, Brown WR, McEwan AR, Rowley PA: Site-specific recombination by phiC31 integrase and other large serine recombinases. Biochem Soc Trans 2010, 38:388-394

24. Ghosh P, Pannunzio NR, Hatfull GF: Synapsis in phage Bxb1 integration: selection mechanism for the correct pair of recombination sites. $J \mathrm{Mol}$ Biol 2005, 349:331-348.

25. Adams V, Lucet IS, Tynan FE, Chiarezza M, Howarth PM, Kim J, Rossjohn J, Lyras D, Rood J: Two distinct regions of the large serine recombinase TnpX are required for DNA binding and biological function. Mol Microbiol 2006, 60:591-601.

26. Thorpe HM, Smith MC: In vitro site-specific integration of bacteriophage DNA catalyzed by a recombinase of the resolvase/invertase family. Proc Natl Acad Sci U S A 1998, 95:5505-5510.

27. Bibb LA, Hancox MI, Hatfull GF: Integration and excision by the large serine recombinase phiRv1 integrase. Mol Microbiol 2005, 55:1896-1910.

28. McEwan AR, Rowley PA, Smith MC: DNA binding and synapsis by the large C-terminal domain of phiC31 integrase. Nucleic Acids Res 2009, 37:4764-4773

29. Lucet IS, Tynan FE, Adams V, Rossjohn J, Lyras D, Rood Jl: Identification of the structural and functional domains of the large serine recombinase TnpX from Clostridium perfringens. J Biol Chem 2005, 280:2503-2511.

30. Lauer P, Chow MY, Loessner MJ, Portnoy DA, Calendar R: Construction, characterization, and use of two Listeria monocytogenes site-specific phage integration vectors. J Bacterio/ 2002, 184:4177-4186.

31. Keravala A, Groth AC, Jarrahian S, Thyagarajan B, Hoyt JJ, Kirby PJ, Calos MP: A diversity of serine phage integrases mediate site-specific recombination in mammalian cells. Mol Genet Genomics 2006, 276:135-146.

32. McEwan AR, Raab A, Kelly SM, Feldmann J, Smith MC: Zinc is essential for high-affinity DNA binding and recombinase activity of phiC31 integrase Nucleic Acids Res 2011, 39:6137-6147.

33. Zhang $L$, Ou $X$, Zhao G, Ding $X$ : Highly efficient in vitro site-specific recombination system based on Streptomyces phage phiBT1 integrase. J Bacteriol 2008, 190:6392-6397.

34. Morita K, Yamamoto T, Fusada N, Komatsu M, Ikeda H, Hirano N, Takahashi H: In vitro characterization of the site-specific recombination system based on actinophage TG1 integrase. Mol Genet Genomics 2009, 282:607-616.

35. Ghosh P, Kim Al, Hatfull GF: The orientation of mycobacteriophage Bxb1 integration is solely dependent on the central dinucleotide of attP and attB. Mol Cell 2003, 12:1101-1111.

36. Neidhardt FC, Bloch PL, Smith DF: Culture medium for enterobacteria. J Bacteriol 1974, 119:736-747.

37. Manning GS: The molecular theory of polyelectrolyte solutions with applications to the electrostatic properties of polynucleotides. Q Rev Biophys 1978, 11:179-246.

38. Flashner $Y$, Gralla JD: DNA dynamic flexibility and protein recognition: differential stimulation by bacterial histone-like protein HU. Cell 1988, 54:713-721.

39. Vologodskii AV, Cozzarelli NR: Conformational and thermodynamic properties of supercoiled DNA. Annu Rev Biophys Biomo/ Struct 1994, 23:609-643.

40. Haykinson MJ, Johnson LM, Soong J, Johnson RC: The Hin dimer interface is critical for Fis-mediated activation of the catalytic steps of site-specific DNA inversion. Curr Biol 1996, 6:163-177.

41. Heiss JK, Sanders ER, Johnson RC: Intrasubunit and intersubunit interactions controlling assembly of active synaptic complexes during Hin-catalyzed DNA recombination. J Mol Biol 2011, 411:744-764.

42. Rowley PA, Smith MC, Younger E: A motif in the C-terminal domain of phiC31 integrase controls the directionality of recombination. Nucleic Acids Res 2008, 36:3879-3891.

43. Sanderson MR, Freemont PS, Rice PA, Goldman A, Hatfull GF, Grindley ND, Steitz TA: The crystal structure of the catalytic domain of the site-specific recombination enzyme gamma delta resolvase at $2.7 \AA$ resolution. Cell 1990, 63:1323-1329

44. Yang W, Steitz TA: Crystal structure of the site-specific recombinase gammadelta resolvase complexed with a 34 bp cleavage site. Cell 1995, 82:193-207.
45. Johnson RC, Bruist MF: Intermediates in Hin-mediated DNA inversion: a role for Fis and the recombinational enhancer in the strand exchange reaction. EMBO J 1989, 8:1581-1590.

46. Breuner A, Brondsted L, Hammer K: Novel organization of genes involved in prophage excision identified in the temperate lactococcal bacteriophage TP901-1. J Bacterio/ 1999, 181:7291-7297.

47. Ghosh P, Wasil LR, Hatfull GF: Control of phage Bxb1 excision by a novel recombination directionality factor. PLoS Biol 2006, 4:e186.

48. Khaleel $T$, Younger $E$, McEwan AR, Varghese AS, Smith MC: A phage protein that binds phiC31 integrase to switch its directionality. Mol Microbiol 2011, 80:1450-1463.

49. Breuner A, Brondsted L, Hammer K: Resolvase-like recombination performed by the TP901-1 integrase. Microbiology 2001, 147:2051-2063.

50. Groth AC, Olivares EC, Thyagarajan B, Calos MP: A phage integrase directs efficient site-specific integration in human cells. Proc Natl Acad Sci U S A 2000, 97:5995-6000

51. Miura T, Hosaka Y, Yan-Zhuo Y, Nishizawa T, Asayama M, Takahashi H, Shirai $M$ : In vivo and in vitro characterization of site-specific recombination of actinophage R4 integrase. J Gen Appl Microbiol 2011, 57:45-57.

52. Gupta M, Till R, Smith MC: Sequences in attB that affect the ability of phiC31 integrase to synapse and to activate DNA cleavage. Nucleic Acids Res 2007, 35:3407-3419.

doi:10.1186/1759-8753-4-2

Cite this article as: Mandali et al:: The site-specific integration reaction of Listeria phage A118 integrase, a serine recombinase. Mobile DNA 2013 $4: 2$

\section{Submit your next manuscript to BioMed Central and take full advantage of:}

- Convenient online submission

- Thorough peer review

- No space constraints or color figure charges

- Immediate publication on acceptance

- Inclusion in PubMed, CAS, Scopus and Google Scholar

- Research which is freely available for redistribution 